\title{
A Probabilistic Topology Unaware TDMA Medium Access Control Policy for Ad Hoc Environments ${ }^{\star}$
}

\author{
Konstantinos Oikonomou ${ }^{1}$ and Ioannis Stavrakakis ${ }^{2}$ \\ 1 INTRACOM S.A., Development Programmes Department, \\ 19.5 Markopoulou Avenue, Paiania 19002 \\ Athens, Greece \\ Tel: +30210 6677023, Fax: +302106671312 \\ okon@intracom.gr \\ 2 University of Athens, \\ Department of Informatics \& Telecommunications \\ Panepistimiopolis, Ilissia 15784 \\ Athens, Greece \\ Tel: +30 2107275343 , Fax: +30 2107275333 \\ istavrak@di.uoa.gr
}

\begin{abstract}
The design of an efficient Medium Access Control (MAC) is challenging in ad-hoc networks where users can enter, leave or move inside the network without any need for prior configuration. Chlamtac and Farago have proposed a topology unaware TDMA-based scheme, suitable for ad-hoc networks, while Ju and Li have proposed an enhanced version that maximizes the minimum guaranteed throughput. Both approaches consider a deterministic policy for the utilization of the assigned scheduling time slots. In this work it is shown that this deterministic policy fails to utilize non-assigned slots that would result in collision-free transmissions even under heavy traffic conditions. A simple probabilistic policy is proposed, capable of utilizing the non-assigned slots according to an access probability, fixed for all users in the network. An analytical study establishes the conditions under which the probability of success for a specific transmission under the probabilistic policy, is higher than that under the deterministic. The dependence of both policies on the topology density is shown and a simple topology density metric is introduced as well. Simulation results show that there exists a suitable range of values for the access probability for which the probabilistic policy outperforms the deterministic and show how this range is affected by the variations of the topology density.
\end{abstract}

\section{Introduction}

Nowadays, the user demand for modern applications anytime and anywhere cannot be fulfilled by the traditional networks (wired, optical, infrastructure-

* This work has been supported in parts by the IST program under contract IST2001-32686 (BroadWay). 
based mobile, etc.). These networks are infrastructure-based and require prior configuration in order for a user to be allowed to use the network resources. On the other hand, ad-hoc networks are infrastructureless and a node can enter, leave or move inside the network without any need for prior configuration. The infrastructureless nature of the ad-hoc networks provides flexibility to the user but at the same time introduces new challenges. The idiosyncrasies of the adhoc networks make the design of an efficient Medium Access Control (MAC) a challenging problem.

Several MAC protocols have been proposed for ad-hoc networks. CSMA/CA has been employed as the main mechanism, [1], [2], [3], whereas many others have employed the Ready-To-Send/Clear-To-Send (RTS/CTS) mechanism, [4], [5], in addition to CSMA/CA, to avoid the hidden/exposed terminal problem.

TDMA-based MAC protocols have also been proposed for ad-hoc networks. S-TDMA - proposed by Kleinrock and Nelson, [6], is capable of providing collision-free scheduling based on the exploitation of noninterfering transmissions in the network. Chlamtac and Farago, [7], have proposed an original TDMAbased scheme for topology transparent scheduling. Their scheme exploits the mathematical properties of polynomials with coefficients from finite Galois fields, to randomly assign scheduling time slot sets to each node of the network. Collisions are allowed, but it is guaranteed that for each node at least one time slot in a frame would be collision-free, [7]. Ju and Li, [8], have proposed another scheme that maximizes the minimum guaranteed throughput. However, certain factors, such as the topology density and the particular random assignment of the polynomials, have not been taken into account.

In this paper the general approach proposed in [7] and [8] is considered and the idea of allowing the nodes to utilize (according to a common access probability) scheduling slots not originally assigned (according to the rules in [7], [8]) to them, is introduced. As it is shown in this paper, the proposed policy achieves a higher throughput under certain conditions (that are studied here), when the benefit of utilizing otherwise idle slots outweighs the loss due to collisions induced by the introduced controlled interference.

In Section 2 a general ad-hoc network is described and some key definitions are introduced. The proposed policy (to be referred to as the Probabilistic Pol$i c y)$ is motivated and introduced in Section 3; the one introduced in [7], [8] is also described and is referred to as the Deterministic Policy. In Section 4 the case of a specific transmission between two given neighbor nodes, is considered. A study is presented establishing the conditions (topology density and polynomial assignment) under which the Probabilistic Policy achieves higher success probability, for the specific transmission; it is also shown that the set of two-hop neighbor nodes influence the probability of success, for a specific transmission. In Section 5 the system throughput is discussed and the motivation for the introduction of a topology density metric and a categorization of topologies is presented in Section 6. The simulation results, presented in Section 7, show that there exists a range of values of the access probability used to utilize probabilistically the otherwise idle slots, that result in a higher throughput. Furthermore, the simula- 
tion results show that this range of values decreases almost exponentially, as the density of the topology increases, as it is expected from the analysis in Section 4. Section 8 presents the conclusions.

\section{System Definition}

An ad-hoc network may be viewed as a time varying multihop network and may be described in terms of a graph $G(V, E)$, where $V$ denotes the set of nodes and $E$ the set of links between the nodes at a given time instance. Let $|X|$ denote the number of elements in set $X$ and let $N=|V|$ denote the number of nodes in the network. Let $S_{u}$ denote the set of neighbors of node $u, u \in V$. These are the nodes $v$ to which a direct transmission from node $u$ (transmission $u \rightarrow v$ ) is possible. Let $D$ denote the maximum number of neighbors for a node; clearly $\left|S_{u}\right| \leq D, \forall u \in V$.

Suppose that a node $u$ wants to transmit to a particular neighbor node $v$ in a particular time slot $i$. In order for the transmission $u \rightarrow v$ to be successful, two conditions should be satisfied. First, node $v$ should not transmit in the particular time slot $i$, or equivalently, no transmission $v \rightarrow \psi, \forall \psi \in S_{v}$ should take place in time slot $i$. Second, no neighbor of $v$ - except $u$ - should transmit in time slot $i$, or equivalently, no transmission $\zeta \rightarrow \chi, \forall \zeta \in S_{v}-\{u\}$ and $\chi \in S_{\zeta}$, should take place in time slot $i$. Consequently, transmission $u \rightarrow v$ is corrupted it time slot $i$ if at least one transmission $\chi \rightarrow \psi, \chi \in S_{v} \cup\{v\}-\{u\}$ and $\psi \in S_{\chi}$, takes place in time slot $i$.

The transmission(s) that corrupts transmission $u \rightarrow v$ may or may not be successful itself. Specifically, in the presence of transmission $u \rightarrow v$, transmission $\chi \rightarrow \psi, \chi \in S_{v} \cup\{v\}-\{u\}$ and $\psi \in S_{\chi} \cap\left(S_{u} \cup\{u\}\right)$, is corrupted. If $\psi \in$ $S_{\chi}-\left(S_{\chi} \cap\left(S_{u} \cup\{u\}\right)\right)$, then transmission $\chi \rightarrow \psi$ is not affected by transmission $u \rightarrow v$.

\section{Scheduling Policies}

Under the Deterministic Policy, [7], [8], each node $u \in V$ is randomly assigned a unique polynomial $f_{u}$ of degree $k$ with coefficients from a finite Galois field of order $q(G F(q))$. Polynomial $f_{u}$ is represented as $f_{u}(x)=\sum_{i=0}^{k} a_{i} x^{i}(\bmod q)$ [8], where $a_{i} \in\{0,1,2, \ldots, q-1\}$; parameters $q$ and $k$ are calculated based on $N$ and $D$, according to the algorithm presented either in [7] or [8]. For both algorithms it is satisfied that $k \geq 1$ and $q>k D$ or $q \geq k D+1$ ( $k$ and $D$ are integers). The access scheme considered is a TDMA scheme with a frame consisted of $q^{2}$ time slots. If the frame is divided into $q$ subframes $s$ of size $q$, then the time slot assigned to node $u$ in subframe $s,(s=0,1, \ldots, q-1)$ is given by $f_{u}(s) \bmod q[8]$. Let the set of time slots assigned to node $u$ be denoted as $\Omega_{u}$. Consequently, $\left|\Omega_{u}\right|=q$. The deterministic transmission policy, [7], [8], is the following. 
The Deterministic Policy: Each node $u$ transmits in a slot $i$ only if $i \in \Omega_{u}$, provided that it has data to transmit.

Depending on the particular random assignment of the polynomials, it is possible that two nodes be assigned overlapping time slots (i.e., $\Omega_{u} \cap \Omega_{v} \neq \emptyset$ ). Let $C_{u \rightarrow v}$ be the set of overlapping time slots between those assigned to node $u$ and those assigned to any node $\chi \in S_{v} \cup\{v\}-\{u\}$. $C_{u \rightarrow v}$ is given by (1).

$$
C_{u \rightarrow v}=\Omega_{u} \cap\left(\bigcup_{\chi \in S_{v} \cup\{v\}-\{u\}} \Omega_{\chi}\right) .
$$

Let $R_{u \rightarrow v}$ denote the set of time slots $i, i \notin \Omega_{u}$, over which transmission $u \rightarrow v$ would be successful. Equivalently, $R_{u \rightarrow v}$ contains those slots not included in set $\bigcup_{\chi \in S_{v} \cup\{v\}} \Omega_{\chi}$. Consequently,

$$
\left|R_{u \rightarrow v}\right|=q^{2}-\left|\bigcup_{\chi \in S_{v} \cup\{v\}} \Omega_{\chi}\right| .
$$

$R_{u \rightarrow v}$ is the set of non-assigned eligible time slots for transmission $u \rightarrow v$, that if used by transmission $u \rightarrow v$, the probability of success for the particular transmission could be increased. The increased probability of success for transmission $u \rightarrow v$ does not necessarily increase the average probability of success of all transmissions in the network (throughput); the presence of transmission $u \rightarrow v$ in a slot $i, i \notin \Omega_{u}$, may corrupt another, otherwise successful, transmission $\chi \rightarrow \psi$. Then, transmission $\chi \rightarrow \psi$ will not be a successful one, even though $u \rightarrow v$ will be.

Theorem 1. $\left|R_{u \rightarrow v}\right|$ is greater than or equal to $q(k-1) D$.

Proof. Notice that $\left|\bigcup_{\chi \in S_{v} \cup\{v\}} \Omega_{\chi}\right| \leq\left(\left|S_{v}\right|+1\right) q$, since $\left|\Omega_{\chi}\right|=q, \forall \chi \in V$. From Equation (2) it is concluded that $\left|R_{u \rightarrow v}\right| \geq q^{2}-\left(\left|S_{v}\right|+1\right) q$, or $\left|R_{u \rightarrow v}\right| \geq$ $q\left(q-\left|S_{v}\right|-1\right)$. Given that $D \geq\left|S_{v}\right|,\left|R_{u \rightarrow v}\right| \geq q(q-D-1)$. Since $q \geq k D+1$ (see [7], [8]), $q-D-1 \geq(k-1) D$. Consequently, $\left|R_{u \rightarrow v}\right| \geq q(k-1) D$.

From Theorem 1 it is obvious that for $k>1,\left|R_{u \rightarrow v}\right|>q D$. Consequently, the number of non-assigned eligible slots may be quite significant for the cases where $k>1$ (this case corresponds to large networks, [8]). Even for the case where $k=1,\left|R_{u \rightarrow v}\right| \geq 0$, that is, $\left|R_{u \rightarrow v}\right|$ can still be greater than zero. For those nodes for which the set of overlapping slots is not the largest possible (i.e., $\left.\left|\bigcup_{\chi \in S_{v} \cup\{v\}} \Omega_{\chi}\right|<\left(\left|S_{v}\right|+1\right) q\right),\left|R_{u \rightarrow v}\right|$ is greater than zero, even for $k=1$. Furthermore, if the neighborhood of node $v$ is not dense, or $\left|S_{v}\right|$ is small compared to $D$, then $\left|R_{u \rightarrow v}\right|$ is even higher.

In general, the use of slots $i, i \in R_{u \rightarrow v}$, may increase the average number of successful transmissions, as long as $R_{u \rightarrow v}$ is determined and time slots $i \in$ $R_{u \rightarrow v}$ are used efficiently. The determination of $R_{u \rightarrow v}$ requires the existence of a 
mechanism for the extraction of sets $\Omega_{\chi}, \forall \chi \in S_{v}$. In addition, the efficient use of slots in $R_{u \rightarrow v}$ by node $u$, requires further coordination and control exchange with neighbor nodes $\chi$, whose transmissions $\chi \rightarrow \psi$, with $R_{\chi \rightarrow \psi} \cap R_{u \rightarrow v} \neq \emptyset$, may utilize the same slots in $R_{\chi \rightarrow \psi} \cap R_{u \rightarrow v}$ and corrupt either transmission $u \rightarrow v$ or $\chi \rightarrow \psi$, or both.

Moreover, under non-heavy traffic conditions, there exist a number of idle slots, in addition to those in $R_{u \rightarrow v}$, not used by the node they are assigned to. In order to use all non-assigned time slots without the need for further coordination among the nodes, the following probabilistic transmission policy is proposed.

The Probabilistic Policy: Each node $u$ always transmits in slot $i$ if $i \in \Omega_{u}$ and transmits with probability $p$ in slot $i$ if $i \notin \Omega_{u}$, provided it has data to transmit.

The Probabilistic Policy does not require specific topology information (e.g., knowledge of $R_{u \rightarrow v}$, etc.) and, thus, induces no additional control overhead. The access probability $p$ is a simple parameter common for all nodes. Under the Probabilistic Policy, all slots $i \notin \Omega_{u}$ are potentially utilized by node $u$ : both, those in $R_{u \rightarrow v}$, for a given transmission $u \rightarrow v$, as well as those not in $\Omega_{u} \cup$ $R_{u \rightarrow v}$ that may be left by neighboring nodes under non-heavy traffic conditions. On the other hand, the probabilistic transmission attempts induce interference to otherwise collision-free transmissions. The following section establishes the conditions under which the loss due to the induced interference is more than compensated for by the utilization of the non-assigned time slots.

\section{Specific Transmission Analysis}

In this section both policies are analyzed for a specific transmission (transmission $u \rightarrow v)$. The analysis assumes heavy traffic conditions; that is, there is always data available for transmission at each node, for every time slot. Let $P_{i, u \rightarrow v}$ denote the probability that transmission $u \rightarrow v$ in slot $i$ is successful. Let $P_{u \rightarrow v}$ be the average probability over a frame for transmission $u \rightarrow v$ to be successful during a time slot. That is,

$$
P_{u \rightarrow v}=\frac{1}{q^{2}} \sum_{i=1}^{q^{2}} P_{i, u \rightarrow v}
$$

where $q^{2}$ is the frame size, in time slots.

Under the Deterministic Policy, $P_{i, u \rightarrow v}=0, \forall i \notin \Omega_{u}$. For $i \in \Omega_{u}$ there are two distinct cases: for $i \in C_{u \rightarrow v}, P_{i, u \rightarrow v}=0$, while for $i \notin C_{u \rightarrow v}, P_{i, u \rightarrow v}=1$ (note the if $i \in C_{u \rightarrow v}$ then $i \in \Omega_{u}$ as well). Since $\left|\Omega_{u}\right|=q$, it is evident that under the Deterministic Policy the average over a frame probability of success for transmission $u \rightarrow v$ (denoted by $P_{D, u \rightarrow v}$ ), is given by

$$
P_{D, u \rightarrow v}=\frac{q-\left|C_{u \rightarrow v}\right|}{q^{2}} .
$$

Under the Probabilistic Policy, it is evident that $P_{i, u \rightarrow v}=0$, for $i \in C_{u \rightarrow v}$, as well as for $i \notin \Omega_{u}$ and $i \notin R_{u \rightarrow v}$. On the other hand, $P_{i, u \rightarrow v}=(1-p)^{\left|S_{v}\right|}$, 
for $i \in \Omega_{u}$ and $i \notin C_{u \rightarrow v}$, whereas $P_{i, u \rightarrow v}=p(1-p)^{\left|S_{v}\right|}$, for $i \in R_{u \rightarrow v}$ (note that if $i \in R_{u \rightarrow v}$ then $i \notin \Omega_{u}$ ). Consequently, $P_{i, u \rightarrow v}=(1-p)^{\left|S_{v}\right|}$ for $q-$ $\left|C_{u \rightarrow v}\right|$ time slots, while $P_{i, u \rightarrow v}=p(1-p)^{\left|S_{v}\right|}$ for $\left|R_{u \rightarrow v}\right|$ time slots. As a result, under the Probabilistic Policy the average over a frame probability of success for transmission $u \rightarrow v$ (denoted by $P_{P, u \rightarrow v}$ ), is given by

$$
P_{P, u \rightarrow v}=\frac{q-\left|C_{u \rightarrow v}\right|+p\left|R_{u \rightarrow v}\right|}{q^{2}}(1-p)^{\left|S_{v}\right|} .
$$

The term $\frac{q-\left|C_{u \rightarrow v}\right|}{q^{2}}(1-p)^{\left|S_{v}\right|}$ is equal to $P_{D, u \rightarrow v}$, decreased by the factor $(1-$ $p)^{\left|S_{v}\right|}$ that is due to the interference introduced by the probabilistic transmission attempts. The term $\frac{p\left|R_{u \rightarrow v}\right|}{q^{2}}(1-p)^{\left|S_{v}\right|}$ is the gain due to the use of the nonassigned eligible slots $R_{u \rightarrow v}$. The aforementioned reduction of $P_{D, u \rightarrow v}$ due to the interference, is possible to be more than compensated for by the gain due to the use of the non-assigned eligible slots, resulting in $P_{P, u \rightarrow v}>P_{D, u \rightarrow v}$. This is investigated in the sequel.

It is evident that $P_{P, u \rightarrow v}=P_{D, u \rightarrow v}$, when $p=0$. For $p>0, P_{P, u \rightarrow v}$ may or may not be greater than $P_{D, u \rightarrow v}$, depending on the values of $p, q,\left|S_{v}\right|,\left|R_{u \rightarrow v}\right|$ and $\left|C_{u \rightarrow v}\right|$. The scope of the following analysis is to determine the conditions under which $P_{P, u \rightarrow v}>P_{D, u \rightarrow v}$.

Theorem 2. $P_{P, u \rightarrow v}=P_{D, u \rightarrow v}$ for $p=0$ and $P_{P, u \rightarrow v}<P_{D, u \rightarrow v}$ for $1 \geq p>0$, provided that $\left|R_{u \rightarrow v}\right| \leq\left(q-\left|C_{u \rightarrow v}\right|\right)\left|S_{v}\right|$.

The proof of Theorem 2 can be seen in Appendix 1.

Theorem 3. Provided that $\left|R_{u \rightarrow v}\right|>\left(q-\left|C_{u \rightarrow v}\right|\right)\left|S_{v}\right|$ is satisfied, $P_{P, u \rightarrow v}>$ $P_{D, u \rightarrow v}$ for $p \in\left(0, p_{\max , u \rightarrow v}\right)$, for some $0<p_{\max , u \rightarrow v}<1$.

The proof of Theorem 3 can be seen in Appendix 3.

According to Appendix 3 there exists a maximum value for $P_{P, u \rightarrow v}$ for a particular value of $p \in\left(0, p_{\max , u \rightarrow v}\right)\left(\equiv p_{0, u \rightarrow v}\right)$. In Appendix 2 it is shown that there exists a value $p_{s, u \rightarrow v}$, such that $p_{0, u \rightarrow v}<p_{s, u \rightarrow v}<1$, for which the second derivative of $P_{P, u \rightarrow v}$ with respect to $p$ is zero.

It should be noted that $p_{\max , u \rightarrow v}$ is not easy to be calculated analytically from Equation (5). On the other hand, it can be calculated using numerical methods, such as the well-known Newton-Raphson method.

\section{On the System Throughput}

The analysis presented so far has established the conditions for which the probability of success under the Probabilistic Policy is higher than that under the Deterministic Policy, for a specific transmission. Since the same value of $p$ is assumed to be adopted for all nodes under the Probabilistic Policy it is possible that this common value results in different comparative performance under the Deterministic and the Probabilistic Policies, for different transmissions. First, it 
may be that for some transmissions Theorem 2 holds and thus the Probabilistic Policy can never outperform the Deterministic one. Second, it may be that for some transmissions $\chi \rightarrow \psi$ Theorem 3 holds but the common $p$ is outside the range $\left(0, p_{\max , \chi \rightarrow \psi}\right)$ and thus the Probabilistic Policy induces a lower probability of success. Finally, for some transmissions $\chi \rightarrow \psi$ Theorem 3 may hold and $p$ is within the range $\left(0, p_{\max , \chi \rightarrow \psi}\right)$ and thus the Probabilistic Policy outperforms the Deterministic one. From the aforementioned three cases it is clear that the system throughput (averaged over all transmissions) under the Probabilistic Policy (denoted by $P_{P, s u c c}$ ) may or may not outperform that under the Deterministic Policy (denoted by $P_{D, \text { succ }}$ ) for a given value of $p$.

The following theorem shows that for large networks $(k>1,[8])\left|R_{u \rightarrow v}\right| \geq$ $\left(q-\left|C_{u \rightarrow v}\right|\right)\left|S_{v}\right|$ holds for every transmission $u \rightarrow v$. For $k=1$ the condition also holds for any transmission $u \rightarrow v$ for which $\left|S_{v}\right| \leq D / 2$.

Theorem 4. For $k>1,\left|R_{u \rightarrow v}\right| \geq\left(q-\left|C_{u \rightarrow v}\right|\right)\left|S_{v}\right|$, for every transmission $u \rightarrow v$. For $k=1\left|R_{u \rightarrow v}\right| \geq\left(q-\left|C_{u \rightarrow v}\right|\right)\left|S_{v}\right|$, provided that $\left|S_{v}\right| \leq D / 2$.

Proof. From Theorem 1 and for any transmission $u \rightarrow v$, it is concluded that $\left|R_{u \rightarrow v}\right| \geq q D$, for $k>1$. Since $q \geq q-\left|C_{u \rightarrow v}\right|$ and $\left|S_{v}\right| \leq D$, it is concluded that $\left(q-\left|C_{u \rightarrow v}\right|\right)\left|S_{v}\right| \leq q D$, and, consequently, $\left|R_{u \rightarrow v}\right| \geq\left(q-\left|C_{u \rightarrow v}\right|\right)\left|S_{v}\right|$ holds for $k>1$.

In [7], [8] it is established that $q \geq D+1$ for $k=1$. From Theorem 1 and for any transmission $u \rightarrow v,\left|R_{u \rightarrow v}\right| \geq q\left(q-\left|S_{v}\right|-1\right)$ or $\left|R_{u \rightarrow v}\right| \geq\left(q-\left|C_{u \rightarrow v}\right|\right)(q-$ $\left.\left|S_{v}\right|-1\right)$, since $q \geq q-\left|C_{u \rightarrow v}\right|$. To show that $\left|R_{u \rightarrow v}\right| \geq\left(q-\left|C_{u \rightarrow v}\right|\right)\left|S_{v}\right|$ it suffices to show that $q-\left|S_{v}\right|-1 \geq\left|S_{v}\right|$ or $q \geq 2\left|S_{v}\right|+1$. Since $q \geq D+1$, it suffices to show that $D+1 \geq 2\left|S_{v}\right|+1$ or $\left|S_{v}\right| \leq D / 2$.

From theorems 2, 3 and 4 , it is shown that for $k>1$ there exists a range of values of $p$ such that $P_{P, \chi \rightarrow \psi} \geq P_{D, \chi \rightarrow \psi}$, for any transmissions $\chi \rightarrow \psi$. Consequently, for $k>1$, there exists a range of values for $p$ such that $P_{P, s u c c} \geq$ $P_{D, \text { succ }}$.

The analysis presented in the previous section (as well as in Theorem 4) has shown an obvious connection between the number of the neighbor nodes of node $v$ and the probability of success for transmission $u \rightarrow v$. From equations (1) and (2) it is concluded that $\left|C_{u \rightarrow v}\right|$ increases and $\left|R_{u \rightarrow v}\right|$ decreases as $\left|S_{v}\right|$ increases. Under the Deterministic Policy (see Equation (4)) the probability of success for transmission $u \rightarrow v$ decreases linearly as $\left|C_{u \rightarrow v}\right|$ increases (as $\left|S_{v}\right|$ increases). Under the Probabilistic Policy (see Equation (5)) as $\left|S_{v}\right|$ increases the probability of success: (a) decreases linearly as $\left|C_{u \rightarrow v}\right|$ increases; (b) increases linearly as $\left|R_{u \rightarrow v}\right|$ decreases; and (c) decreases exponentially $\left(\operatorname{term}(1-p)^{\left|S_{v}\right|}\right)$ as $\left|S_{v}\right|$ increases. Thus, it seems that an increase in $\left|S_{v}\right|$ has a more negative impact on the probability of success under the Probabilistic Policy than under the Deterministic. Consequently, it is expected that: (a) the Probabilistic Policy (with access probability $p$ ) outperforms the Deterministic Policy for a certain topology $G(V, E)$ with parameters $N$ and $D$ and low topology density, while the reverse can be true for the same parameters $N$ and $D$ and access probability $p$ but a 
higher topology density; (b) the value of $p$ that results in a higher probability of success for the Probabilistic Policy decreases, to reduce the impact of the interference caused by the increased number of probabilistic attempts by the increased number of neighbors. The maximum system throughput achieved under the Probabilistic Policy under some $p$ is expected to decrease as the topology density increases.

From the above discussion, it is evident that the topology density affects strongly the probability of success under the Probabilistic Policy. In the next section, a topology density metric is introduced to be used for the categorization of each topology $G(V, E)$ for a given pair $N, D$. In Section 7 , the relation between the range of suitable values of $p$ (range of values for which $P_{P, \text { succ }} \geq P_{D, \text { succ }}$ ) and the topology density metric is investigated as well as the relation between the maximum value of $P_{P, \text { succ }}$ and the particular metric.

\section{Topology Density Analysis}

It has been shown that a specific transmission $u \rightarrow v$ is influenced (is corrupted or successful) by the transmission set $\Phi_{u \rightarrow v} \cup \Theta_{u \rightarrow v}$. Consequently, node $v$ as well as any node $\chi \in S_{v}-\{u\}$ influence transmission $u \rightarrow v$. Let the set of nodes $S_{u}$ be referred to as the set of one-hop neighbor nodes of node $u$ with respect to transmission $u \rightarrow v$, the set of nodes $S_{v}-\{u\}, \forall v \in S_{u}$, be referred to as the set of two-hop neighbor nodes of node $u$ and the union of both previous sets to be referred to as the aggregate two-hop set of neighbor nodes of node $u$ with respect to transmission $u \rightarrow v$. Consequently, the aggregate two-hop set of a node influences the transmissions of that particular node. Based on this observation, the topology density is defined as it is presented next.

For a given pair $N$ and $D$ numerous topologies $G(V, E)$ exist. It is possible to categorize these topologies depending on the number of the aggregate two-hop nodes. Let Calculated $(G(V, E))$ denote the total number of aggregate two-hop nodes, for all nodes in the network, calculated for topology $G(V, E)$.

$$
\text { Calculated }(G(V, E))=\sum_{u \in V} \sum_{v \in S_{u}}\left|S_{v}-\{u\}\right|=\sum_{u \in V} \sum_{v \in S_{u}}\left(\left|S_{v}\right|-1\right) .
$$

Let $\operatorname{Upper}(N, D)$ and Lower $(N, D)$ be defined as follows.

$$
\begin{gathered}
\operatorname{Upper}(N, D)=N D^{2}, \\
\operatorname{Lower}(N, D)= \begin{cases}D^{2}+D & N-D=1 \\
D^{2}+D+4 & N-D=2 \\
D^{2}-3 D+4 N-4 & N-D \geq 3 .\end{cases}
\end{gathered}
$$

Theorem 5. For a topology $G(V, E)$ with parameters $N$ and $D$, the following holds: Lower $(N, D) \leq$ Calculated $(G(V, E)) \leq U \operatorname{Uper}(N, D)$. 

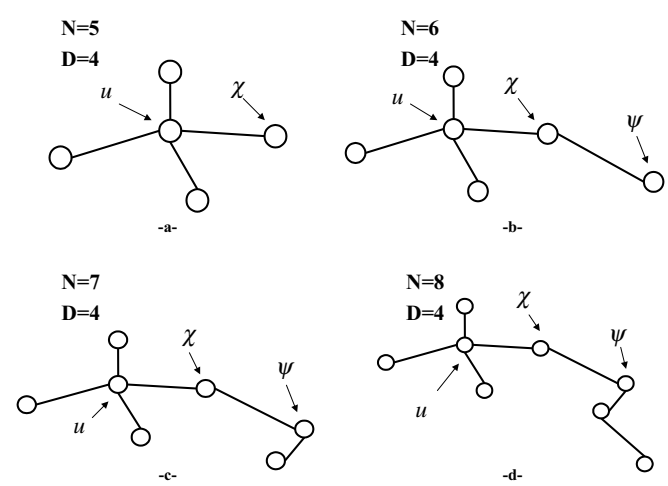

Fig. 1. Least dense topology for $D=4$ and $N=5,6,7,8$ respectively.

Proof. The upper bound for Calculated $(G(V, E))$ is derived by considering the most dense topology $G(V, E)$ for which $N=|V|$. The most dense topology corresponds to the case for which $\left|S_{u}\right|=D, \forall u \in V$. Consequently, for each of the $N$ nodes, there are $D$ one-hop neighbors, and $D(D-1)$ two-hop neighbors. Thus, the total aggregate two-hop nodes in the network are $N(D(D-1)+D)=N D^{2}$. As a result, $\operatorname{Upper}(N, D)=N D^{2}$. Note that in the case in which the most dense topology for a pair $N$ and $D$ is not possible to have $D$ neighbor nodes for every node, $\operatorname{Upper}(N, D)<N D^{2}$ still holds.

The lower bound for Calculated $(G(V, E))$ corresponds to the total number of aggregate two-hop nodes of the least dense topology possible for a given pair $N, D$. The latter is a topology in which there is only one node $u$ with $\left|S_{u}\right|=D$ one-hop neighbor nodes, $D-1$ nodes are one-hop neighbors of $u$ but have no other one-hop neighbor except node $u$, and one node $\chi$ is one-hop neighbor of node $u$ and the initiator of a line of nodes. No loops are present in the topology (see Figure 1).

In case $N-D=1$ (see Figure 1.a for the corresponding least topology) each node other than $u$ has only one one-hop neighbor node; node $u$. Consequently, there are $D$ aggregate two-hop nodes for node $u$, as well as $D$ for the rest $D$ nodes. As a result, $\operatorname{Lower}(D, N)=D^{2}+D$, for $N-D=1$.

In case $N-D=2$ (see Figure 1.b for the corresponding least dense topology) node $\chi$ has another one-hop neighbor node, node $\psi$, expect $u$. Consequently, there are $D+1$ aggregate two-hop nodes for node $\chi$, and 2 for node $\psi$. For node $u$, there are $D+1$ aggregate two-hop nodes, and for each of the $D-1$ remaining nodes (for which their only one-hop neighbor node is $u$ ), $D$ aggregate two-hop nodes. As a result Lower $(D, N)=(D-1) D+(D+1)+(D+1)+2$ or $\operatorname{Lower}(D, N)=D^{2}+D+4$, for $N-D=2$.

In case $N-D \geq 3$ (see Figures 1.c and 1.d for the corresponding least dense topology) node $\chi$ is the initiator of a line of nodes. The number of nodes in this line is equal to $N-D-1$. For the two nodes at the end of the line there is $3+2$ aggregate two-hop nodes, while for each one of the $N-D-3$ nodes there are 4 . 
As a result Lower $(D, N)=(D-1) D+(D+1)+(D+2)+4(N-D-3)+3+2$ or $\operatorname{Lower}(D, N)=D^{2}-3 D+4 N-4$ for $N-D \geq 3$.

Let $W(G(V, E))$ denote a density metric for topology $G(V, E)$ defined as follows;

$$
W(G(V, E))=\frac{\text { Calculated }(G(V, E))-\operatorname{Lower}(N, D)}{U \text { pper }(N, D)-\operatorname{Lower}(N, D)} .
$$

It is evident from Theorem 5 that $0 \leq W(G(V, E)) \leq 1$. For $W(G(V, E))$ close to 1 the number of the aggregate two-hop nodes is high, while for $W(G(V, E))$ close to zero the number of the aggregate two-hop nodes is low.

\section{Simulation Results}

For the simulation purposes four different topology categories are considered. The number of nodes in each topology category is $N=100$, while $D$ is set to 5 , 10, 15 and 20. These four topology categories are denoted as D5N100, D10N100, D15N100 and D20N100 respectively. Parameters $q$ and $k$ are determined according to the algorithm presented in [8]. Time slot sets are assigned randomly to each node, for each particular topology. The particular assignment is kept the same for each topology category throughout the simulations. Different topologies that correspond to different topology density values $W(G(V, E))$ are considered for each topology category.
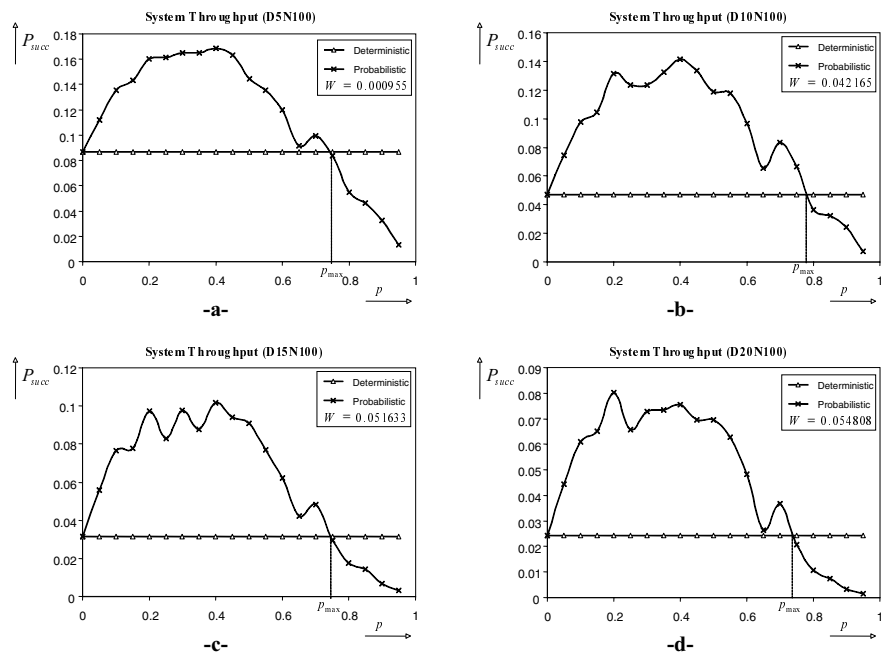

Fig. 2. $P_{\text {succ }}$ under the Deterministic as well as the Probabilistic Policy, for small topology density values. 
Note from Theorem 1 that for $k>1$ the number of non-assigned eligible time slots is expected to be higher for a higher value of $k$ and Theorem 3 always holds as it has been proved in Theorem 4. Consequently, it is expected that for $k>1$ all transmissions will achieve a higher throughput under the Probabilistic Policy for any value of $p$ in the range $\left(0, p_{\max }\right)$, where $p_{\max }$ depends on the specific transmission's environment (see Theorem 3). When $k=1$, the Probabilistic Policy will outperform the Deterministic Policy for those transmission for which $\left|S_{v}\right| \leq D / 2$ and may not for others. That is, the lower the topology density the more transmissions will satisfy the condition $\left|S_{v}\right| \leq D / 2$ and the wider the range of values of $p$ for which the Probabilistic Policy will outperform the Deterministic Policy.

The simulation results presented demonstrate the performance for $k=1$ (the resulting value for $k$ is equal to 1 for the four topology categories, [8]), that is the case that the number of non-assigned eligible time slots is expected to be rather small and, thus, the effectiveness of the Probabilistic Policy rather low.

The simulation results show that: (a) there exists a range of values for $0 \leq$ $p \leq p_{\max }\left(0<p_{\max }<1\right)$ such that $P_{P, \text { succ }} \geq P_{D \text {,succ }}$; (b) this range decreases as the topology density, measured by $W(G(V, E))$, increases as expected, suggesting that the introduced topology density metric $W(G(V, E))$ is an effective one; (c) $P_{P, \text { succ }}$ decreases as $W(G(V, E))$ increases.

Figures 2 and 3 depict simulation results for the system throughput $\left(P_{\text {succ }}\right)$, under both the Deterministic and the Probabilistic Policies, as a function of the access probability $p$. In Figure 2, $W(G(V, E))$ is close to zero, while in Figure 3, $W(G(V, E))$ is as high as possible (close to 0.8). In all three sets of simulations it can be observed that the system throughput achieved under the Deterministic Policy is constant with respect to $p$. Under the Probabilistic Policy there exists a range of values for $p\left(0 \leq p \leq p_{\max }<1\right)$ such that $P_{P, \text { succ }} \geq P_{D, \text { succ }}$.

For values of $p$ close to 1 the throughput is almost zero. This is expected since the interference induced due to the high access probability is rather high; it may also be concluded from Equation (5).

A careful consideration of the results in figures 2 and 3 shows that $p_{\max }$ decreases as $W(G(V, E))$ increases as expected. This trend is shown more clearly in Figure 4, where the values for $p_{\max }$ derived in the simulations are plotted as a function of $W(G(V, E))$. Notice that $p_{\max }$ decreases almost exponentially as $W(G(V, E))$ increases, as expected from the discussion in Section 5 . The results in Figure 4 demonstrate the effectiveness of the introduced topology density metric $W(G(V, E))$ in capturing the topology density and determining the range of probabilities $p$ under the Probabilistic Policy that lead to a higher system throughput than that under the Deterministic Policy. Notice that for a particular value of $W(G(V, E))$, any value of $p$ below the curve results in $P_{P, s u c c} \geq P_{D, \text { succ }}$. Based on the results in Figure 4, one could measure or estimate the value of $W(G(V, E))$ and depending on whether it is low or high select a larger or smaller probability $p$. 

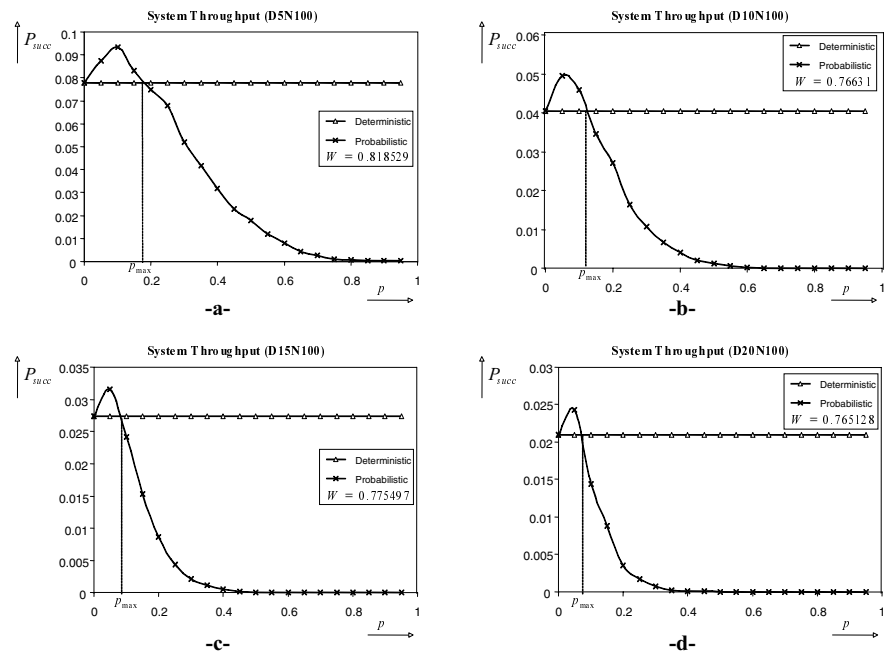

Fig. 3. $P_{\text {succ }}$ under the Deterministic as well as the Probabilistic Policy, for high topology density values.
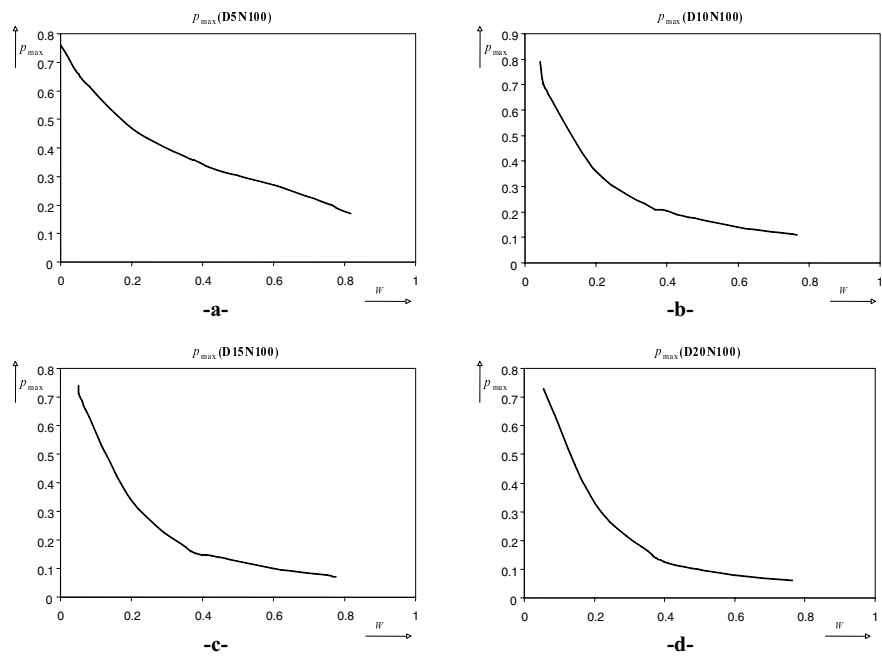

Fig. 4. $p_{\max }$ parameterized by $W(G(V, E))$.

\section{Summary and Conclusions}

In this paper the inherent inefficiencies of the Deterministic (slot assignment) Policy in an ad-hoc network, proposed in [7] and [8], are investigated and the Probabilistic (slot assignment) Policy is introduced in an effort to improve the 
achieved network throughput. The basic idea behind the proposed policy is to use (with some probability $p$ ) slots not assigned to a node under the assignment scheme in [7], [8]. The study in this paper has been carried out under heavy traffic conditions, which are expected to minimize the benefits of the Probabilistic Policy that eventually tries to utilize slots non-assigned to anybody or not used by others.

A common probability $p$ is assumed for all transmissions in the network - as it would practically be the case - and the system throughput is considered, as shaped by all transmissions (which may or may not achieve a higher throughput under the Probabilistic Policy). First, it is shown that the networks for which the assigned polynomials have degree $k>1$ (see [7], [8]) - which is the case for large networks - a nonzero range for the common probability $p$ exists under which the Probabilistic Policy outperforms the Deterministic Policy. Second, for $k=1$ (typically for rather small networks) sufficient conditions are established regarding the two-hop neighborhood under which the conditions for the existence of a range $\left(0, p_{\max }\right)$ of the access probability are satisfied and, thus, such transmissions will achieve a higher throughput under the Probabilistic Policy; thus, such transmissions will contribute a higher value toward the system throughput achieved under the Probabilistic Policy. The latter conditions show that the size of the two-hop neighborhood affects the performance of of a specific transmission (the smaller the size the higher the achieved throughput) and suggest that the system throughput achieved under the Probabilistic Policy is affected by the overall one- and two-hop (aggregate two-hop) neighborhood size of the network. These observations have led to the idea of introducing the topology density metric $W(G(V, E))$ and use it to infer how large the aggregate two-hop neighborhood is for a certain topology. As $W(G(V, E))$ decreases it is expected under the Probabilistic Policy that both the range of effective access probabilities $\left(0, p_{\max }\right)$ and the achieved system throughput will increase.

Simulation results have been derived for four network topology categories (for four pairs $(N, D)$ ) and the for two values of the corresponding value of $W(G(V, E))$ for each one of them; $k=1$ and heavy traffic conditions have been assumed, both of which are expected to induce only a small advantage of the Probabilistic Policy over the Deterministic Policy, compared to the cases of networks with $k>1$ and non-heavy traffic conditions. The derived results have supported the claims and expectations regarding the comparative advantage of the Probabilistic Policy over the Deterministic Policy, as well as the dependence of the former on the introduced topology density metric.

\section{References}

1. IEEE 802.11, "Wireless LAN Medium Access Control (MAC) and Physical Layer (PHY) specifications", Nov. 1997. Draft Supplement to Standard IEEE 802.11, IEEE, New York, January 1999. 
2. V. Bharghavan, A. Demers, S. Shenker, and L. Zhang, "MACAW: A Media Access Protocol for Wireless LAN's", Proceedings of ACM SIGCOMM'94, pp. 212-225, 1994.

3. C.L. Fullmer, J.J. Garcia-Luna-Aceves, "Floor Acquisition Multiple Access (FAMA) for Packet-Radio Networks", Proceedings of ACM SIGCOMM'95, pp. 262-273, 1995.

4. P. Karn, "MACA - A new channel access method for packet radio", in ARRL/CRRL Amateur Radio 9th Computer Networking Conference, pp. 134-140, 1990.

5. J. Deng and Z. J. Haas, "Busy Tone Multiple Access (DBTMA): A New Medium Access Control for Packet Radio Networks," in IEEE ICUPC'98, Florence, Italy, October 5-9, 1998.

6. R. Nelson, L. Kleinrock, "Spatial TDMA, A collision-free Multihop Channel Access Protocol", IEEE Transactions on Communications, Vol. COM-33, No. 9, September 1985.

7. I. Chlamtac and A. Farago, "Making Transmission Schedules Immune to Topology Changes in Multi-Hop Packet Radio Networks", IEEE/ACM Trans. on Networking, 2:23-29, 1994.

8. J.-H. Ju and V. O. K. Li, "An Optimal Topology-Transparent Scheduling Method in Multihop Packet Radio Networks", IEEE/ACM Trans. on Networking, 6:298-306, 1998.

\section{Appendices}

\section{Appendix 1 Proof of Theorem 2}

From Equation (5) it is concluded that $P_{P, u \rightarrow v}=P_{D, u \rightarrow v}$ for $p=0$.

The first derivative of $P_{P, u \rightarrow v}$ with respect to $p$ is calculated in Appendix 4 . For $\left|R_{u \rightarrow v}\right| \leq\left(q-\left|C_{u \rightarrow v}\right|\right)\left|S_{v}\right|$, the first derivative is zero for two values of $p$ : $p=0$ and $p=1$. For any other value $p \in(0,1)$ the first derivative is always negative (see Appendix 4) and therefore, the global maximum corresponds to $p=0\left(P_{P, u \rightarrow v}=P_{D, u \rightarrow v}\right)$ whereas, the global minimum corresponds to $p=1$ $\left(P_{P, u \rightarrow v}=0\right)$. Consequently, for any value of $p \in(0,1], P_{P, u \rightarrow v}<P_{D, u \rightarrow v}$.

Appendix 2 On the Existence of $p_{s, u \rightarrow v}$

$\frac{d^{2} g(p)}{d^{2} p}=0$ for $p=1$ and $p=\frac{2\left|R_{u \rightarrow v}\right|-\left(q-\left|C_{u \rightarrow v}\right|\right)\left(\left|S_{v}\right|-1\right)}{\left|R_{u \rightarrow v}\right|\left(\left|S_{v}\right|+1\right)} \equiv p_{s, u \rightarrow v}$. Since $0<p \leq 1$ ( 0 is not included since it is the case for which $P_{P, u \rightarrow v}=P_{D, u \rightarrow v}$ ), in order for $p_{s, u \rightarrow v}$ to be a valid root it is required that $0<p_{s, u \rightarrow v} \leq 1$. $p_{s, u \rightarrow v}>0$ when $2\left|R_{u \rightarrow v}\right|>\left(q-\left|C_{u \rightarrow v}\right|\right)\left(\left|S_{v}\right|-1\right)$. Note that $p_{s, u \rightarrow v} \leq 1$, when $p_{s, u \rightarrow v}>0$. Notice that $2\left|R_{u \rightarrow v}\right|>\left(q-\left|C_{u \rightarrow v}\right|\right)\left(\left|S_{v}\right|-1\right)$ is always met if $\left|R_{u \rightarrow v}\right|>(q-$ $\left.\left|C_{u \rightarrow v}\right|\right)\left|S_{v}\right|$ and, in this case, $p_{s, u \rightarrow v}>p_{0, u \rightarrow v}$. To show the latter it suffices to show that $\frac{2\left|R_{u \rightarrow v}\right|-\left(q-\left|C_{u \rightarrow v}\right|\right)\left(\left|S_{v}\right|-1\right)}{\left|R_{u \rightarrow v}\right|\left(\left|S_{v}\right|+1\right)}>\frac{\left|R_{u \rightarrow v}\right|-\left(q-\left|C_{u \rightarrow v}\right|\right)\left|S_{v}\right|}{\left|R_{u \rightarrow v}\right|\left(\left|S_{v}\right|+1\right)}$ or $2\left|R_{u \rightarrow v}\right|-(q-$ $\left.\left|C_{u \rightarrow v}\right|\right)\left(\left|S_{v}\right|-1\right)>\left|R_{u \rightarrow v}\right|-\left(q-\left|C_{u \rightarrow v}\right|\right)\left|S_{v}\right|$ or $2\left|R_{u \rightarrow v}\right|-\left(q-\left|C_{u \rightarrow v}\right|\right)\left|S_{v}\right|+$ $q-\left|C_{u \rightarrow v}\right|>\left|R_{u \rightarrow v}\right|-\left(q-\left|C_{u \rightarrow v}\right|\right)\left|S_{v}\right|$ or $2\left|R_{u \rightarrow v}\right|+q-\left|C_{u \rightarrow v}\right|>\left|R_{u \rightarrow v}\right|$ or $\left|R_{u \rightarrow v}\right|+q-\left|C_{u \rightarrow v}\right|>0$. The latter always holds. 


\section{Appendix 3 Proof of Theorem 3}

From Equation (5) it is concluded that $P_{P, u \rightarrow v}=P_{D, u \rightarrow v}$ for $p=0$ and $P_{P, u \rightarrow v}=$ 0 for $p=1$. Consequently, the range of values for which $P_{P, u \rightarrow v}>P_{D, u \rightarrow v}$ includes neither 0 nor 1 .

For $0<p<1$ and $\left|R_{u \rightarrow v}\right|>\left(q-\left|C_{u \rightarrow v}\right|\right)\left|S_{v}\right|$ the first derivative of $P_{P, u \rightarrow v}$, with respect to $p$, is zero (see Appendix 4), when $p=\frac{\left|R_{u \rightarrow v}\right|-\left(q-\left|C_{u \rightarrow v}\right|\right)\left|S_{v}\right|}{\left|R_{u \rightarrow v}\right|\left(\left|S_{v}\right|+1\right)}$ $\left(\equiv p_{0, u \rightarrow v}\right)$. For $p=p_{0, u \rightarrow v}$ (the first derivative is zero) the second derivative is negative (see Appendix 4). Consequently, $p_{0, u \rightarrow v}$ corresponds to a maximum value for $P_{P, u \rightarrow v}$. For every value $p, 0<p \leq p_{0, u \rightarrow v}$, the first derivative is always positive and consequently, $P_{P, u \rightarrow v}>P_{D, u \rightarrow v}$.

On the other hand, for every value $p_{0, u \rightarrow v}<p<1$ the first derivative is always negative. For $p \rightarrow 1, P_{P, u \rightarrow v} \rightarrow 0$ and given that $P_{P, u \rightarrow v}$ is a continuous function of $p$, there exists a value $p_{0, u \rightarrow v}<p<1$, such that $P_{P, u \rightarrow v}=P_{D, u \rightarrow v}$. Let $p_{\max , u \rightarrow v}$ denote that value of $p$.

Finally, it is evident that for any value $p \in\left(0, p_{\max , u \rightarrow v}\right), P_{P, u \rightarrow v}>P_{D, u \rightarrow v}$, provided that $\left|R_{u \rightarrow v}\right|>\left(q-\left|C_{u \rightarrow v}\right|\right)\left|S_{v}\right|$.

Appendix 4 Complement of Proofs of Theorems 2 and 3

For convenience, the following function $g(p)$ is considered

$$
g(p)=\left(q-\left|C_{u \rightarrow v}\right|+\left|R_{u \rightarrow v}\right| p\right)(1-p)^{\left|S_{v}\right|}=P_{p, u \rightarrow v} q^{2} .
$$

The first and second derivative of $g(p)$ with respect to $p$ are given by:

$$
\begin{aligned}
& \frac{d g(p)}{d p}=\left(\left|R_{u \rightarrow v}\right|-\left(q-\left|C_{u \rightarrow v}\right|\right)\left|S_{v}\right|-\left|R_{u \rightarrow v}\right|\left(\left|S_{v}\right|+1\right) p\right)(1-p)^{\left|S_{v}\right|-1} . \\
& \frac{d^{2} g(p)}{d^{2} p}=-\left|S_{v}\right|\left(2\left|R_{u \rightarrow v}\right|-\left(q-\left|C_{u \rightarrow v}\right|\right)\left(\left|S_{v}\right|-1\right)-\left|R_{u \rightarrow v}\right|\left(\left|S_{v}\right|+1\right) p\right)(1-p)^{\left|S_{v}\right|-2} .
\end{aligned}
$$

$\frac{d g(p)}{d p}=0$ for $p=1$ and $p=\frac{\left|R_{u \rightarrow v}\right|-\left(q-\left|C_{u \rightarrow v}\right|\right)\left|S_{v}\right|}{\left|R_{u \rightarrow v}\right|\left(\left|S_{v}\right|+1\right)} \equiv p_{0, u \rightarrow v}$. Since $0<p \leq 1$, in order for $p_{0, u \rightarrow v}$ to be a valid root it is required that $0<p_{0, u \rightarrow v} \leq 1 . p_{0, u \rightarrow v}>0$ when $\left|R_{u \rightarrow v}\right|>\left(q-\left|C_{u \rightarrow v}\right|\right)\left|S_{v}\right|$. Note that $p_{0, u \rightarrow v} \leq 1$, when $p_{0, u \rightarrow v}>0$.

If $\left|R_{u \rightarrow v}\right|<\left(q-\left|C_{u \rightarrow v}\right|\right)\left|S_{v}\right|$ then $\frac{d g(p)}{d p}<0$ for any value of $p$. Therefore the maximum value for $g(p)$ is assumed for $p=0$ and it is $g(0)=q-\left|C_{u \rightarrow v}\right|$.

If $\left|R_{u \rightarrow v}\right|>\left(q-\left|C_{u \rightarrow v}\right|\right)\left|S_{v}\right|$ then for $p=p_{0, u \rightarrow v}$ the second derivative is equal to

$$
\left.\frac{d^{2} g(p)}{d^{2} p}\right|_{p=p_{0, u \rightarrow v}}=-\left|S_{v}\right|\left(q-\left|C_{u \rightarrow v}\right|+\left|R_{u \rightarrow v}\right|\right)(1-p)^{\left|S_{v}\right|-2} .
$$

Since $\left(q-\left|C_{u \rightarrow v}\right|\right)+\left|R_{u \rightarrow v}\right|>0$ and $(1-p)^{\left|S_{v}\right|-2}>0$ for $p=p_{0, u \rightarrow v}$, it is concluded that $\left.\frac{d^{2} g(p)}{d^{2} p}\right|_{p=p_{0, u \rightarrow v}}<0$. As a result $g(p)$ assumes a maximum value at $p_{0, u \rightarrow v}$. 